21st Particles and Nuclei International Conference (PANIC 2017)

International Journal of Modern Physics: Conference Series

Vol. 46 (2018) 1860083 (4 pages)

(C) The Author(s)

DOI: $10.1142 / \mathrm{S} 2010194518600832$

\title{
D-Wave Heavy Baryons from QCD Sum Rules
}

\author{
Qiang Mao \\ Department of Electrical and Electronic Engineering, Suzhou University, Suzhou 234000, China \\ maoqiang@ahszu.edu.cn \\ Hua-Xing Chen \\ School of Physics and Beijing Key Laboratory of Advanced Nuclear Materials \\ and Physics, Beihang University, Beijing 100191, China \\ hxchen@buaa.edu.cn \\ Atsushi Hosaka \\ Research Center for Nuclear Physics, Osaka University, Ibaraki 567-0047, Japan \\ hosaka@rcnp.osaka-u.ac.jp \\ Xiang Liu \\ School of Physical Science and Technology, Lanzhou University, Lanzhou 730000, China \\ xiangliu@lzu.edu.cn \\ Shi-Lin Zhu \\ School of Physics and State Key Laboratory of Nuclear Physics \\ and Technology, Peking University, Beijing 100871, China \\ zhusl@pku.edu.cn
}

Published 3 May 2018

\begin{abstract}
We study the $D$-wave heavy baryons using the method of QCD sum rules in the framework of heavy quark effective theory. Our results suggest that the $\Lambda_{c}(2860), \Lambda_{c}(2880)$, $\Xi_{c}(3055)$ and $\Xi_{c}(3080)$ complete two $D$-wave $S U(3)$ flavor $\overline{\mathbf{3}}_{F}$ charmed baryon doublets of $J^{P}=3 / 2^{+}$and $5 / 2^{+}$.
\end{abstract}

Keywords: Excited heavy baryons; QCD sum rule; heavy quark effective theory.

\section{Introduction}

In the past years important experimental progresses have been made in the field of heavy baryons ${ }^{1}$. All the $1 S$ charmed baryons have been well established, and the

This is an Open Access article published by World Scientific Publishing Company. It is distributed under the terms of the Creative Commons Attribution 4.0 (CC-BY) License. Further distribution of this work is permitted, provided the original work is properly cited. 
$1 P$ states $\Lambda_{c}(2595), \Lambda_{c}(2625), \Xi_{c}(2790)$ and $\Xi_{c}(2815)$ have also been well observed and complete two $S U(3)$ flavor $\overline{\mathbf{3}}_{F}$ multiplets of $J^{P}=1 / 2^{-}$and $3 / 2^{-}$. Besides them, there still exist many higher states, and various phenomenological methods and models were applied to investigate them. We refer to reviews ${ }^{2,3,4,5}$ for their recent progress.

In this paper we shall concentrate on the $\Lambda_{c}(2860), \Lambda_{c}(2880), \Xi_{c}(3055)$ and $\Xi_{c}(3080)^{1,6}$. To study them, we shall use the method of QCD sum rules ${ }^{7,8}$ in the framework of heavy quark effective theory ${ }^{9,10,11}$. This method has also been applied to study the $S$ - and $P$-wave heavy baryons ${ }^{12,13,14}$.

\section{QCD Sum Rule Analyses}

In Refs. 15, 16 we have systematically constructed all the $D$-wave heavy baryon interpolating fields. Here we briefly explain our notations: $J_{j, P, F, j_{l}, s_{l}, \rho-\lambda}^{\alpha_{1} \cdots \alpha_{j-1 / 2}}$ denotes the $D$-wave heavy baryon field belonging to the baryon multiplet $\left[F, j_{l}, s_{l}, \rho-\lambda\right]$, where $j, P$, and $F$ are its total angular momentum, parity and $S U(3)$ flavor representation $\left(\overline{\mathbf{3}}_{F}\right.$ or $\left.\mathbf{6}_{F}\right)$, respectively; $j_{l}$ and $s_{l}$ are the total angular momentum and spin angular momentum of its light components, respectively. There are three types: $\rho \rho$-type $\left(l_{\rho}=2\right.$ and $\left.l_{\lambda}=0\right), \lambda \lambda$-type $\left(l_{\rho}=0\right.$ and $\left.l_{\lambda}=2\right)$ and $\rho \lambda$-type $\left(l_{\rho}=1\right.$ and $\left.l_{\lambda}=1\right)$, where $l_{\rho}$ is the orbital angular momentum between the two light quarks, and $l_{\lambda}$ is the orbital angular momentum between the heavy quark and the two-light-quark system.

We summarize all the possible configurations of the $D$-wave $(L=2)$ charmed baryons in Fig. 1, where $\mathbf{A}$ and $\mathbf{S}$ denote the structure to be antisymmetric and symmetric, respectively. The explicit forms of $J_{j, P, F, j_{l}, s_{l}, \rho-\lambda}^{\alpha_{1} \cdots \alpha_{j-1 / 2}}$ have been given in Eqs. (2-19) of Ref. 15. Here we use the current $J_{3 / 2,+, \Lambda_{c}, 2,0, \lambda \lambda}^{\alpha}$ as an example:

$$
\begin{aligned}
J_{3 / 2,+, \Lambda_{c}, 2,0, \lambda \lambda}^{\alpha}(x)= & \epsilon_{a b c}\left(\left[\mathcal{D}_{\mu_{1}}^{t} \mathcal{D}_{\mu_{2}}^{t} u^{a T}(x)\right] \mathbb{C} \gamma_{5} d^{b}(x)+\right. \\
& \left.2\left[\mathcal{D}_{\mu_{1}}^{t} u^{a T}(x)\right] \mathbb{C} \gamma_{5}\left[\mathcal{D}_{\mu_{2}}^{t} d^{b}(x)\right]+u^{a T}(x) \mathbb{C} \gamma_{5}\left[\mathcal{D}_{\mu_{1}}^{t} \mathcal{D}_{\mu_{2}}^{t} d^{b}(x)\right]\right) \\
\times & \left(\frac{1}{2} g_{t}^{\mu_{1} \alpha} g_{t}^{\mu_{2} \mu_{4}}+\frac{1}{2} g_{t}^{\mu_{2} \alpha} g_{t}^{\mu_{1} \mu_{4}}-\frac{1}{3} g_{t}^{\mu_{1} \mu_{2}} g_{t}^{\mu_{4} \alpha}\right) \times \gamma_{\mu_{4}}^{t} \gamma_{5} h_{v}^{c}(x) .
\end{aligned}
$$

We use it to perform QCD sum rule analyses. We refer to Refs. 12-17 for details, but simply list here the equation to evaluate the mass of the heavy baryon belonging to the multiplet $\left[F, j_{l}, s_{l}, \rho-\lambda\right]$ :

$$
m_{j, P, F, j_{l}, s_{l}, \rho-\lambda}=m_{Q}+\bar{\Lambda}_{F, j_{l}, s_{l}, \rho-\lambda}+\delta m_{j, P, F, j_{l}, s_{l}, \rho-\lambda},
$$

where $\bar{\Lambda}_{F, j_{l}, s_{l}, \rho-\lambda}=\bar{\Lambda}_{\left|j_{l}-1 / 2\right|, P, F, j_{l}, s_{l}, \rho-\lambda}=\bar{\Lambda}_{j_{l}+1 / 2, P, F, j_{l}, s_{l}, \rho-\lambda}$ is the sum rule result obtained at the leading order, and $\delta m_{j, P, F, j_{l}, s_{l}, \rho-\lambda}$ is the sum rule result obtained at the $\mathcal{O}\left(1 / m_{Q}\right)$ order:

$$
\delta m_{j, P, F, j_{l}, s_{l}, \rho-\lambda}=-\frac{1}{4 m_{Q}}\left(K_{F, j_{l}, s_{l}, \rho-\lambda}+d_{M} C_{m a g} \Sigma_{F, j_{l}, s_{l}, \rho-\lambda}\right) .
$$


$[\rho \rho] l_{\rho}=2(\mathbf{S}), l_{\lambda}=0, \overline{\mathbf{3}}_{\mathbf{C}}(\mathbf{A})$

$$
\begin{aligned}
& \overline{\mathbf{3}}_{\mathbf{F}}(\mathbf{A}): L=2 \otimes s_{l}=0(\mathbf{A})-j_{l}=2: \Lambda_{\mathrm{c} 2}\left(\frac{3}{2}^{+}, \frac{5}{2}^{+}\right) \quad \Xi_{\mathrm{c} 2}\left(\frac{3}{2}^{+}, \frac{5}{2}^{+}\right) \quad(\rho \rho-\mathrm{a})\left[\overline{\mathbf{3}}_{\mathbf{F}}, 2,0, \rho \rho\right] \\
& \mathbf{6}_{\mathbf{F}}(\mathbf{S}): L=2 \otimes s_{l}=1(\mathbf{S})\left\{\begin{array}{llll}
j_{l}=1: \Sigma_{\mathrm{c} 1}\left(\frac{1}{2}^{+}, \frac{3}{2}^{+}\right) & \Xi_{\mathrm{c} 1}\left(\frac{1}{2}^{+}, \frac{3}{2}^{+}\right) & \Omega_{\mathrm{c} 1}\left(\frac{1}{2}^{+}, \frac{3}{2}^{+}\right) & (\rho \rho-\mathrm{b})\left[\mathbf{6}_{\mathbf{F}}, 1,1, \rho \rho\right] \\
j_{l}=2: \Sigma_{\mathrm{c} 2}\left(\frac{3}{2}^{+}, \frac{5}{2}^{+}\right) & \Xi_{\mathrm{c} 2}\left(\frac{3}{2}^{+}, \frac{5^{+}}{2}\right) & \Omega_{\mathrm{c} 2}\left(\frac{3}{2}^{+}, \frac{5}{2}^{+}\right) & (\rho \rho-\mathrm{c})\left[\mathbf{6}_{\mathbf{F}}, 2,1, \rho \rho\right] \\
j_{l}=3: \Sigma_{\mathrm{c} 3}\left(\frac{5}{2}^{+}, \frac{7}{2}^{+}\right) & \Xi_{\mathrm{c} 3}\left(\frac{5}{2}^{+}, \frac{7}{2}^{+}\right) & \Omega_{\mathrm{c} 3}\left(\frac{5}{2}^{+}, \frac{7}{2}^{+}\right) & (\rho \rho-\mathrm{d})\left[\mathbf{6}_{\mathbf{F}}, 3,1, \rho \rho\right]
\end{array}\right.
\end{aligned}
$$

$[\lambda \lambda] l_{\rho}=0(\mathbf{S}), l_{\lambda}=2, \overline{\mathbf{3}}_{\mathbf{C}}(\mathbf{A})$

$$
\begin{aligned}
& \overline{\mathbf{3}}_{\mathbf{F}}(\mathbf{A}): L=2 \otimes s_{l}=0(\mathbf{A})-j_{l}=2: \Lambda_{\mathrm{c} 2}\left(\frac{3}{2}^{+}, \frac{5}{2}^{+}\right) \quad \Xi_{\mathrm{c} 2}\left(\frac{3}{2}^{+}, \frac{5}{2}^{+}\right) \quad(\lambda \lambda-\mathrm{a})\left[\overline{\mathbf{3}}_{\mathbf{F}}, 2,0, \lambda \lambda\right] \\
& \mathbf{6}_{\mathbf{F}}(\mathbf{S}): L=2 \otimes s_{l}=1(\mathbf{S})\left\{\begin{array}{llll}
j_{l}=1: \Sigma_{\mathrm{c} 1}\left(\frac{1}{2}^{+}, \frac{3}{2}^{+}\right) & \Xi_{\mathrm{c} 1}\left(\frac{1}{2}^{+}, \frac{3^{+}}{2}\right) & \Omega_{\mathrm{c} 1}\left(\frac{1}{2}^{+}, \frac{3}{2}^{+}\right) & (\lambda \lambda-\mathrm{b})\left[\mathbf{6}_{\mathbf{F}}, 1,1, \lambda \lambda\right] \\
j_{l}=2: \Sigma_{\mathrm{c} 2}\left(\frac{3}{2}^{+}, \frac{5}{2}^{+}\right) & \Xi_{\mathrm{c} 2}\left(\frac{3}{2}^{+}, \frac{5}{2}^{+}\right) & \Omega_{\mathrm{c} 2}\left(\frac{3}{2}^{+}, \frac{5}{2}^{+}\right) & (\lambda \lambda-\mathrm{c})\left[\mathbf{6}_{\mathbf{F}}, 2,1, \lambda \lambda\right] \\
j_{l}=3: \Sigma_{\mathrm{c} 3}\left(\frac{5}{2}^{+}, \frac{7}{2}^{+}\right) & \Xi_{\mathrm{c} 3}\left(\frac{5}{2}^{+}, \frac{7}{2}^{+}\right) & \Omega_{\mathrm{c} 3}\left(\frac{5}{2}^{+}, \frac{7}{2}^{+}\right) & (\lambda \lambda-\mathrm{d})\left[\mathbf{6}_{\mathbf{F}}, 3,1, \lambda \lambda\right]
\end{array}\right.
\end{aligned}
$$

$[\rho \lambda] l_{\rho}=1(\mathbf{A}), l_{\lambda}=1, \overline{\mathbf{3}}_{\mathbf{C}}(\mathbf{A})$

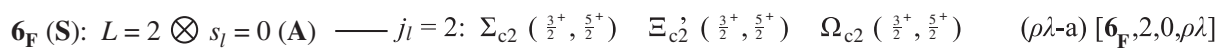

$$
\begin{aligned}
& \overline{\mathbf{3}}_{\mathbf{F}}(\mathbf{A}): L=2 \otimes s_{l}=1(\mathbf{S})\left\{\begin{array}{lll}
j_{l}=1: \Lambda_{\mathrm{c} 1}\left(\frac{1}{2}^{+}, \frac{3}{2}^{+}\right) & \Xi_{\mathrm{c} 1}\left(\frac{1}{2}^{+}, \frac{3}{2}^{+}\right) & (\rho \lambda-\mathrm{b})\left[\overline{\mathbf{3}}_{\mathbf{F}}, 1,1, \rho \lambda\right] \\
j_{l}=2: \Lambda_{\mathrm{c} 2}\left(\frac{3}{2}^{+}, \frac{5}{2}^{+}\right) & \Xi_{\mathrm{c} 2}\left(\frac{3}{2}^{+}, \frac{5}{2}^{+}\right) & (\rho \lambda-\mathrm{c})\left[\overline{\mathbf{3}}_{\mathbf{F}}, 2,1, \rho \lambda\right] \\
j_{l}=3: \Lambda_{\mathrm{c} 3}\left(\frac{5}{2}^{+}, \frac{7}{2}^{+}\right) & \Xi_{\mathrm{c} 3}\left(\frac{5}{2}^{+}, \frac{7}{2}^{+}\right) & (\rho \lambda-\mathrm{d})\left[\overline{\mathbf{3}}_{\mathbf{F}}, 3,1, \rho \lambda\right]
\end{array}\right.
\end{aligned}
$$

$L=0 / 1$ : we do not study these two cases in this paper.

Fig. 1. $D$-wave charmed baryons.

Still use the current $J_{3 / 2,+, \Lambda_{c}, 2,0, \lambda \lambda}^{\alpha}$ as an example. We use it to perform QCD sum rule analyses and calculate $\bar{\Lambda}_{\Lambda_{c}, 2,0, \lambda \lambda}, K_{\Lambda_{c}, 2,0, \lambda \lambda}$ and $\Sigma_{\Lambda_{c}, 2,0, \lambda \lambda}$, from which we obtain the following numerical results for the baryon multiplet $\left[\Lambda_{c}\left(\overline{\mathbf{3}}_{F}\right), 2,0, \lambda \lambda\right]$ :

$$
\begin{aligned}
m_{\Lambda_{c}\left(3 / 2^{+}\right)} & =2.81_{-0.18}^{+0.33} \mathrm{GeV}, \\
m_{\Lambda_{c}\left(5 / 2^{+}\right)} & =2.84_{-0.20}^{+0.37} \mathrm{GeV}, \\
m_{\Lambda_{c}\left(5 / 2^{+}\right)}-m_{\Lambda_{c}\left(3 / 2^{+}\right)} & =28_{-24}^{+45} \mathrm{MeV},
\end{aligned}
$$

Similarly, we obtain the following numerical results for the baryon multiplet $\left[\Xi_{c}\left(\overline{\mathbf{3}}_{F}\right), 2,0, \lambda \lambda\right]$ :

$$
\begin{aligned}
m_{\Xi_{c}\left(3 / 2^{+}\right)} & =3.04_{-0.15}^{+0.15} \mathrm{GeV}, \\
m_{\Xi_{c}\left(5 / 2^{+}\right)} & =3.05_{-0.16}^{+0.15} \mathrm{GeV}, \\
m_{\Xi_{c}\left(5 / 2^{+}\right)}-m_{\Xi_{c}\left(3 / 2^{+}\right)} & =15_{-13}^{+16} \mathrm{MeV} .
\end{aligned}
$$

The above values are consistent with the experimental masses of the $\Lambda_{c}(2860)$, $\Lambda_{c}(2880), \Xi_{c}(3055)$, and $\Xi_{c}(3080)^{1,6}$. 


\section{Summary and Discussions}

In this paper we have studied the $D$-wave charmed baryons using the method of QCD sum rules within the heavy quark effective theory. We have calculated their masses up to the order $\mathcal{O}\left(1 / m_{Q}\right)$ with large theoretical uncertainty, and we have also calculated their mass splittings within the same doublet with much less theoretical uncertainty.

Our results suggest that the $\Lambda_{c}(2860), \Lambda_{c}(2880), \Xi_{c}(3055)$ and $\Xi_{c}(3080)$ complete two $D$-wave $S U(3)$ flavor $\overline{\mathbf{3}}_{F}$ charmed baryon doublets $\left[\overline{\mathbf{3}}_{F}, 2,0, \lambda \lambda\right]$ of $J^{P}=$ $3 / 2^{+}$and $5 / 2^{+}$. We propose to further investigate their decay properties in order to study the structure of heavy baryons in detail.

\section{Acknowledgments}

This project is supported by the National Natural Science Foundation of China under Grants No. 11722540, No. 11475015, No. 11375024, No. 11222547, No. 11175073, No. 11575008, and No. 11621131001; the 973 program; the Ministry of Education of China (SRFDP under Grant No. 20120211110002 and the Fundamental Research Funds for the Central Universities); and the Key Natural Science Research Program of Anhui Educational Committee (Grant No. KJ2016A774). A.H. is supported by Grants-in-Aid for Scientific Research (Grants No. JP17K05441(C)).

\section{References}

1. Particle Data Group (C. Patrignani et al.), Chin. Phys. C 40, 100001 (2016).

2. H. X. Chen, W. Chen, X. Liu, Y. R. Liu and S. L. Zhu, Rept. Prog. Phys. 80, 076201 (2017).

3. H. Y. Cheng, Front. Phys. (Beijing) 10, 101406 (2015).

4. E. Klempt and J. M. Richard, Rev. Mod. Phys. 82, 1095 (2010).

5. J. G. Körner, M. Kramer and D. Pirjol, Prog. Part. Nucl. Phys. 33, 787 (1994).

6. LHCb Collab. (R. Aaij et al.), JHEP 1705, 030 (2017).

7. M. A. Shifman, A. I. Vainshtein and V. I. Zakharov, Nucl. Phys. B 147, 385 (1979).

8. L. J. Reinders, H. Rubinstein and S. Yazaki, Phys. Rept. 127, 1 (1985).

9. B. Grinstein, Nucl. Phys. B 339, 253 (1990).

10. E. Eichten and B. R. Hill, Phys. Lett. B 234, 511 (1990).

11. A. F. Falk, H. Georgi, B. Grinstein and M. B. Wise, Nucl. Phys. B 343, 1 (1990).

12. X. Liu, H. X. Chen, Y. R. Liu, A. Hosaka and S. L. Zhu, Phys. Rev. D 77, 014031 (2008).

13. H. X. Chen, W. Chen, Q. Mao, A. Hosaka, X. Liu and S. L. Zhu, Phys. Rev. D 91, $054034(2015)$.

14. Q. Mao, H. X. Chen, W. Chen, A. Hosaka, X. Liu and S. L. Zhu, Phys. Rev. D 92, 114007 (2015).

15. H. X. Chen, Q. Mao, A. Hosaka, X. Liu and S. L. Zhu, Phys. Rev. D 94, 114016 (2016).

16. Q. Mao, H. X. Chen, A. Hosaka, X. Liu and S. L. Zhu, Phys. Rev. D 96, 074021 (2017).

17. H. X. Chen, V. Dmitrasinovic, A. Hosaka, K. Nagata and S. L. Zhu, Phys. Rev. D 78, 054021 (2008). 